\title{
AN EXISTENCE THEOREM FOR SUM AND PRODUCT INTEGRALS
}

\author{
JON C. HELTON
}

Abstract. Functions are from $S \times S$ to $N$, where $S$ and $N$ denote a linearly ordered set and a normed ring, respectively.

THEOREM. If $\{a, b\} \in S \times S, G$ has bounded variation on $\{a, b\}$, $\left\{F_{n}\right\}_{1}^{\infty}$ converges uniformly to a bounded function $F$ on $\{a, b\}$ and either

(1) $x \prod^{y}\left(1+F_{n} G\right)$ and $\int_{a}^{b}\left|1+F_{n} G-\prod\left(1+F_{n} G\right)\right|$

exist for $n=1,2, \cdots$ and each subdivision $\{a, x, y, b\}$ of $\{a, b\}$ and $\left\{\int_{a}^{b}\left|1+F_{n} G-\Gamma\left(1+F_{n} G\right)\right|\right\}_{1}^{\infty}$ converges to zero or

$$
\int_{a}^{b} F_{n} G \text { and } \int_{a}^{b}\left|F_{n} G-\int F_{n} G\right|
$$

exist for $n=1,2, \cdots$ and $\left\{\int_{a}^{b}\left|F_{n} G-\int F_{n} G\right|\right\}_{1}^{\infty}$ converges to zero, then (conclusion) $a \prod^{b}(1+F G), a \prod^{b}(1+|F G|), \quad \int_{a}^{b} F G$ and $\int_{a}^{b}|F G|$ exist. Further, $\int_{a}^{b}\left|1+F G-\prod(1+F G)\right|, \int_{a}^{b}|1+| F G\left|-\prod(1+|F G|)\right|$, $\int_{a}^{b}\left|F G-\int F G\right|$ and $\int_{a}^{b}\left\|F G\left|-\int\right| F G\right\|$ exist and are zero.

All integrals and definitions are of the subdivision-refinement type, and functions are from $S \times S$ to $N$, where $S$ denotes a linearly ordered set and $N$ denotes a ring which has a multiplicative indentity element represented by 1 and has a norm $|\cdot|$ with respect to which $N$ is complete and $|1|=1$. The function $G \in O M^{\circ}$ on $\{a, b\}$ only if ${ }_{x} \Pi^{y}(1+G)$ exists for each subdivision $\{a, x, y, b\}$ of $\{a, b\}$ and $\int_{a}^{b}\left|1+G-\prod(1+G)\right|=0$, and $G \in O A^{\circ}$ on $\{a, b\}$ only if $\int_{a}^{b} G$ exists and $\int_{a}^{b}\left|G-\int G\right|=0$. If $J=\left\{x_{q}\right\}_{0}^{n}$ is a subdivision of $\{a, b\}$, then $G_{q}=G\left(x_{q-1}, x_{q}\right)$. Similarly, if $F_{n}$ is a function, then $F_{n q}=$ $F_{n}\left(x_{q-1}, x_{q}\right)$. Further, we use $J(I), \prod_{J(I)}(1+G)$ and $\sum_{J(I)} G$ to represent $\left\{\left(x_{q-1}, x_{q}\right)\right\}_{1}^{n}, \prod_{1}^{n}\left(1+G_{q}\right)$ and $\sum_{1}^{n} G_{q}$, respectively. In addition, $G$ is bounded on $\{a, b\}, G \in O P^{\circ}$ on $\{a, b\}$, and $G \in O B^{\circ}$ on $\{a, b\}$ only if there exists a subdivision $D$ of $\{a, b\}$ and a number $B$ such that if $J=\left\{x_{q}\right\}_{0}^{n}$ is a refinement of $D$, then

(1) $|G(u)|<B$ for $u \in J(I)$,

(2) $\left|\prod_{i}^{j}\left(1+G_{q}\right)\right|<B$ for $1 \leqq i \leqq j \leqq n$, and

(3) $\sum_{J(I)}|G|<B$,

Received by the editors July 10, 1972.

AMS (MOS) subject classifications (1970). Primary 26A40, 46G99, 46H99.

Key words and phrases. Sum integral, product integral, subdivision-refinement integral, existence, sequence, limit, normed ring, interval function.

(c) American Mathematical Society 1973 
respectively. Note that $O B^{\circ} \subseteq O P^{\circ}$. The statement that the sequence of functions $\left\{F_{n}\right\}_{1}^{\infty}$ converges uniformly to a function $F$ on $\{a, b\}$ means if $\varepsilon>0$ then there exists a subdivision $D$ of $[a, b]$ and a positive integer $N$ such that if $J$ is a refinement of $D$ and $n>N$, then $\left|F(u)-F_{n}(u)\right|<\varepsilon$ for $u \in J(I)$. Consult B. W. Helton [2] and J. S. MacNerney [4] for additional details.

Lemma 1. If $\{a, b\} \in S \times S$ and $F \in O P^{\circ}$ and $G \in O P^{\circ}$ on $\{a, b\}$, then there exists a subdivision $D$ of $\{a, b\}$ and a number $B$ such that if $J=\left\{x_{q}\right\}_{0}^{r}$ is a refinement of $D$ and $1 \leqq i \leqq j \leqq r$, then

$$
\left|\prod_{i}^{j}\left(1+F_{q}\right)-\prod_{i}^{j}\left(1+G_{q}\right)\right| \leqq B \sum_{i}^{j}\left|F_{q}-G_{q}\right|
$$

[2, Theorem 3.2, p. 300].

Lemma 2. If $\{a, b\} \in S \times S, G \in O B^{\circ}$ on $\{a, b\},\left\{F_{n}\right\}_{1}^{\infty}$ converges uniformly to a bounded function $F$ on $\{a, b\}$ and ${ }_{a} \Pi^{b}\left(1+F_{n} G\right)$ exists for $n=$ $1,2, \cdots$, then

(1) $\lim _{n \rightarrow \infty} \Pi^{b}\left(1+F_{n} G\right)$ exists, and

(2) ${ }_{a} \Pi^{b}(1+F G)$ exists and is $\lim _{n \rightarrow \infty} a \Pi^{b}\left(1+F_{n} G\right)$.

Proof. We first show that (1) is true. Let $\varepsilon>0$. There exists a subdivision $D_{1}$ of $\{a, b\}$, a number $B$ and a positive integer $N$ such that if $J$ is a refinement of $D_{1}$ and $n \geqq m>N$, then

(1) $\sum_{J(I)}|G|<B$,

(2) $\left|\prod_{J(I)}\left(1+F_{n} G\right)-\prod_{J(I)}\left(1+F_{m} G\right)\right| \leqq B \sum_{J(I)}\left|F_{n} G-F_{m} G\right|$, and

(3) $\left|F_{n}(u)-F_{m}(u)\right|<\varepsilon / 2 B^{2}$ for $u \in J(I)$.

Let $n \geqq m>N$. There exists a subdivision $D_{2}$ of $\{a, b\}$ such that if $J$ is a refinement of $D_{2}$, then

(1) $\left.\right|_{a} \prod^{b}\left(1+F_{n} G\right)-\prod_{J(I)}\left(1+F_{n} G\right) \mid<\varepsilon / 4$, and

(2) $\left|\prod_{J(I)}\left(1+F_{m} G\right)-{ }_{a} \prod^{b}\left(1+F_{m} G\right)\right|<\varepsilon / 4$.

Let $D=D_{1} \cup D_{2}$, and suppose $J$ is a refinement of $D$. Thus,

$$
\begin{aligned}
\mid a \prod^{b}\left(1+F_{n} G\right)- & { }_{a} \prod^{b}\left(1+F_{m} G\right) \mid \\
& <\left|\prod_{J(I)}\left(1+F_{n} G\right)-\prod_{J(I)}\left(1+F_{m} G\right)\right|+\varepsilon / 2 \\
& \leqq B \sum_{J(I)}\left|F_{n} G-F_{m} G\right|+\varepsilon / 2<B\left(\varepsilon / 2 B^{2}\right) B+\varepsilon / 2=\varepsilon .
\end{aligned}
$$

We now show that $\prod_{a} \Pi^{b}(1+F G)$ exists and is $L$, where

$$
L=\lim _{n \rightarrow \infty} \prod^{b}\left(1+F_{n} G\right) .
$$


Let $\varepsilon>0$. There exists a subdivision $D$ of $\{a, b\}$, a number $B$ and a positive integer $n$ such that if $J$ is a refinement of $D$, then

(1) $\sum_{J(I)}|G|<B$,

(2) $\left|\prod_{J(I)}(1+F G)-\prod_{J(I)}\left(1+F_{n} G\right)\right| \leqq B \sum_{J(I)}\left|F G-F_{n} G\right|$,

(3) $\left|\prod_{J(I)}\left(1+F_{n} G\right)-L\right|<\varepsilon / 2$, and

(4) $\left|F(u)-F_{n}(u)\right|<\varepsilon / 2 B^{2}$ for $u \in J(I)$.

Thus, if $J$ is a refinement of $D$, then

$$
\begin{aligned}
\left|\prod_{J(I)}(1+F G)-L\right| & <\left|\prod_{J(I)}(1+F G)-\prod_{J(I)}\left(1+F_{n} G\right)\right|+\varepsilon / 2 \\
& \leqq B \sum_{J(I)}\left|F G-F_{n} G\right|+\varepsilon / 2<B\left(\varepsilon / 2 B^{2}\right) B+\varepsilon / 2=\varepsilon .
\end{aligned}
$$

Lemma 3. If $\{a, b\} \in S \times S, G \in O B^{\circ}$ on $\{a, b\},\left\{F_{n}\right\}_{1}^{\infty}$ converges uniformly to a bounded function $F$ on $\{a, b\}$ and $\int_{a}^{b} F_{n} G$ exists for $n=1,2, \cdots$, then

(1) $\lim _{n \rightarrow \infty} \int_{a}^{b} F_{n} G$ exists, and

(2) $\int_{a}^{b} F G$ exists and is $\lim _{n \rightarrow \infty} \int_{a}^{b} F_{n} G$.

IndiCATION OF ProOF. Lemma 3 follows by an argument that is similar to the one used for Lemma 2.

Lemma 4. If $\{a, b\} \in S \times S$ and $G \in O B^{\circ}$ on $\{a, b\}$, then the following statements are equivalent:

(1) $G \in O A^{\circ}$ on $\{a, b\}$, and

(2) $G \in O M^{\circ}$ on $\{a, b\}[2$, Theorem 3.4, p. 301].

Lemma 5. If $\{a, b\} \in S \times S$ and $G$ is a real valued function such that $\int_{a}^{b} G$ exists, then $G \in O A^{\circ}$ on $\{a, b\}$.

Lemma 5 is due to A. Kolmogoroff [3, p. 669]. The reader is also referred to results by W. D. L. Appling [1, Theorems 1, 2, p. 155] and B.W. Helton [2, Theorem 4.1, p. 304].

Lemma 6. If $\{a, b\} \in S \times S, G \in O B^{\circ}$ on $\{a, b\}$ and either $G \in O A^{\circ}$ on $\{a, b\}$ or $G \in O M^{\circ}$ on $\{a, b\}$, then $|G| \in O A^{\circ}$ and $O M^{\circ}$ on $\{a, b\}$.

Proof. It follows from Lemma 4 that $G \in O A^{\circ}$ on $\{a, b\}$. There exists a number $B$ such that if $J$ is a subdivision of $\{a, b\}$ then $\sum_{J(I)}\left|\int G\right|<B$. Let $V$ be the set such that $v \in V$ only if there exists a subdivision $J$ of $\{a, b\}$ such that $v=\sum_{J(I)}\left|\int G\right|$. The set $V$ is bounded and thus has a least upper bound $L$.

We now show that $\int_{a}^{b}|G|$ exists and is $L$. Let $\varepsilon>0$. There exists a subdivision $E$ of $\{a, b\}$ such that if $J$ is a refinement of $E$, then

(1) $\sum_{J(I)}\left|\int G\right|>\dot{L}-\varepsilon / 2$, and

(2) $\sum_{J(I)}\left|G-\int G\right|<\varepsilon / 2$. 
Suppose $J$ is a subdivision of $E$. Thus,

$$
\sum_{J(I)}|G| \leqq \sum_{J(I)}\left|\int G\right|+\sum_{J(I)}\left|G-\int G\right|<L+\varepsilon / 2
$$

and

$$
\sum_{J(I)}|G|>\sum_{J(I)}\left|\int G\right|-\sum_{J(I)}\left|G-\int G\right|>(L-\varepsilon / 2)-\varepsilon / 2 .
$$

Therefore,

$$
L-\varepsilon<\sum_{J(I)}|G|<L+\varepsilon .
$$

Hence, $\int_{a}^{b}|G|$ exists. Thus, it follows from Lemma 5 that $|G| \in O A^{\circ}$ on $\{a, b\}$ and from Lemma 4 that $|G| \in O M^{\circ}$ on $\{a, b\}$.

THEOREM. If $\{a, b\} \in S \times S, G \in O B^{\circ}$ on $\{a, b\},\left\{F_{n}\right\}_{1}^{\infty}$ converges uniformly to $a$ bounded function $F$ on $\{a, b\}$ and either

(1) ${ }_{x} \prod^{y}\left(1+F_{n} G\right)$ and $\int_{a}^{b}\left|1+F_{n} G-\prod\left(1+F_{n} G\right)\right|$ exist for $n=1,2, \cdots$ and each subdivision $\{a, x, y, b\}$ of $\{a, b\}$ and $\left\{\int_{a}^{b}\left|1+F_{n} G-\Pi\left(1+F_{n} G\right)\right|\right\}_{1}^{\infty}$ converges to zero, or

(2) $\int_{a}^{b} F_{n} G$ and $\int_{a}^{b}\left|F_{n} G-\int F_{n} G\right|$ exist for $n=1,2, \cdots$ and $\left\{\int_{a}^{b}\left|F_{n} G-\int F_{n} G\right|\right\}_{1}^{\infty}$ converges to zero.

Then $F G \in O M^{\circ}$ and $O A^{\circ}$ on $\{a, b\}$ and $|F G| \in O M^{\circ}$ and $O A^{\circ}$ on $\{a, b\}$.

Proof. Suppose (1) is true. It follows from Lemma 2 that ${ }_{x} \Pi^{y}(1+F G)$ exists for each subdivision $\{a, x, y, b\}$ of $\{a, b\}$. We now show that

$$
\int_{a}^{b}\left|1+F G-\prod(1+F G)\right|=0 \text {. }
$$

Let $\varepsilon>0$. There exists a subdivision $D$ of $\{a, b\}$, a number $B$ and a positive integer $n$ such that if $J=\left\{x_{q}\right\}_{0}^{r}$ is a refinement of $D$ and $J_{q}$ is a subdivision of $\left\{x_{q-1}, x_{q}\right\}$ for $1 \leqq q \leqq r$, then

(1) $\sum_{J(I)}|G|<B$,

(2) $\left|\prod_{J_{q}(I)}\left(1+F_{n} G\right)-\prod_{J_{q}(I)}(1+F G)\right| \leqq B \sum_{J_{q}(I)}\left|F_{n} G-F G\right|$,

(3) $\left|F(u)-F_{n}(u)\right|<\varepsilon / 4 B$ and $\left|F_{n}(u)-F(u)\right|<\varepsilon / 4 B^{2}$ for $u \in J(I)$, and

(4) $\sum_{q=1}^{r}\left|1+F_{n q} G_{q}-\prod_{J_{q}(I)}\left(1+F_{n} G\right)\right|<\varepsilon / 4$.

Let $J=\left\{x_{q}\right\}_{0}^{r}$ be a refinement of $D$. For $q=1,2, \cdots, r$, there exists a subdivision $J_{q}$ of $\left\{x_{q-1}, x_{q}\right\}$ such that

$$
\left|\prod_{J_{q}(I)}(1+F G)-{ }_{x_{q-1}} \prod^{x_{q}}(1+F G)\right|<\varepsilon / 4 r .
$$


Thus,

$$
\begin{aligned}
\sum_{J(I)} \mid 1+F G-\prod & (1+F G) \mid \\
\leqq & \sum_{J(I)}\left|F-F_{n}\right||G|+\sum_{q=1}^{r}\left|1+F_{n q} G_{q}-\prod_{J_{q}(I)}(1+F G)\right| \\
& +\sum_{q=1}^{r}\left|\prod_{J_{q}(I)}(1+F G)-{ }_{x_{q}-1} \prod^{x_{q}}(1+F G)\right| \\
< & (\varepsilon / 4 B) B+\sum_{q=1}^{r}\left|1+F_{n q} G_{q}-\prod_{J_{q}(I)}(1+F G)\right|+r(\varepsilon / 4 r) \\
\leqq & \sum_{q=1}^{r}\left|1+F_{n q} G_{q}-\prod_{J_{q}(I)}\left(1+F_{n} G\right)\right| \\
& +\sum_{q=1}^{r}\left|\prod_{J_{q}(I)}\left(1+F_{n} G\right)-\prod_{J_{q}(I)}(1+F G)\right|+\varepsilon / 2 \\
\leqq & \varepsilon / 4+\sum_{q=1}^{r}\left[B \sum_{J_{q}(I)}\left|F_{n}-F\right||G|\right]+\varepsilon / 2 \\
\leqq & \varepsilon / 4+B\left(\varepsilon / 4 B^{2}\right) B+\varepsilon / 2=\varepsilon .
\end{aligned}
$$

Therefore, $F G \in O M^{\circ}$ on $\{a, b\}$, and hence, $F G \in O A^{\circ}$ on $\{a, b\}$ by Lemma 4. Thus, it follows from Lemma 6 that $|F G| \in O M^{\circ}$ and $O A^{\circ}$ on $\{a, b\}$.

Suppose (2) is true. The desired result follows by using Lemma 3 and an argument similar to the one employed in the preceding paragraph.

The question naturally arises as to whether or not there exist functions and sequences satisfying the conditions in the hypothesis of the Theorem. We now supply an example which was suggested by a result of Appling [1, Theorem $2(1 \rightarrow 2)$, pp. 155-156] on real Hilbert spaces. We consider the set of infinite diagonal matrices with bounded elements and $|M|=$ lub $\left|m_{i j}\right|$. For each positive integer $p$, let $A_{p}$ be the infinite diagonal matrix such that $a_{p p}=1$ and $a_{q q}=0$ if $q \neq p$. Let $A=\bigcup_{p=1}^{\infty} A_{p}$. Let $f$ be a reversible function from the rational numbers contained in $(0,1)$ to $A$. There exists a function $G$ of subintervals of $[0,1]$ such that if $[u, v]$ is a subinterval of $[0,1]$ then there exists a rational number $r$ contained in $[u, v]$ such that

$$
G(u, v)=(v-u) f(r) .
$$

For each positive integer $n$, let $F_{n}(u, v)$ be the diagonal matrix $B_{n}$ such that $b_{q q}=1 / n$. Thus, if $[u, v]$ is a subinterval of $[0,1]$, then

$$
\int^{v} F_{n} G=0 \quad \text { and } \quad \prod_{u}^{v}\left(1+F_{n} G\right)=1 .
$$


Hence,

$$
\int_{0}^{1}\left|F_{n} G-\int F_{n} G\right|=\int_{0}^{1}\left|F_{n} G\right|=1 / n
$$

and

$$
\int_{0}^{1}\left|1+F_{n} G-\prod\left(1+F_{n} G\right)\right|=\int_{0}^{1}\left|1+F_{n} G-1\right|=\int_{0}^{1}\left|F_{n} G\right|=1 / n .
$$

\section{BIBLIOGRAPHY}

1. W. D. L. Appling, Interval functions and real Hilbert spaces, Rend. Circ. Mat. Palermo (2) 11 (1962), 154-156. MR 27 \#4040.

2. B. W. Helton, Integral equations and product integrals, Pacific J. Math. 16 (1966), 297-322. MR 32 \#6167.

3. A. Kolmogoroff, Untersuchungen über den Integralbegriff, Math. Ann. 103 (1930), 654-696.

4. J. S. MacNerney, Integral equations and semigroups, Illinois J. Math. 7 (1963), 148-173. MR 26 \#1726.

Department of Mathematics, Arizona State University, Tempe, Arizona 85281 